\title{
DIMENSÕES DA IMITAÇÃO ENTRE EMPRESAS: UM ESTUDO NA INDÚSTRIA DE TRANSFORMAÇÃO BRASILEIRA
}

\author{
DIMENSIONS OF THE IMITATION AMONG ENTERPRISES: A STUDY IN THE BRAZILIAN MANUFACTURING INDUSTRY \\ DIMENSIONES DE LA IMITACIÓN ENTRE EMPRESAS: UN ESTUDIO EN LA INDUSTRIA DE TRANSFORMACIÓN BRASILEÑA
}

\section{RESUMO}

O objetivo deste trabalho foi identificar as dimensões do construto imitação entre empresas e propor um procedimento operacional para sua mensuração nos setores empresariais. A imitação foi conceituada como cópia de competências. Sua operacionalização foi feita pela facilidade de compreendê-las. Colheram-se as percepções de 112 gestores de 14 setores da economia brasileira no Estado de São Paulo. Utilizou-se a base de dados da Federação da Indústria do estado de São Paulo (Fiesp). As dimensões subjacentes ao construto foram identificadas pela análise fatorial exploratória. Os resultados indicam que a imitação em um setor tende a ser maior quanto menos se realizam atividades de pesquisa e desenvolvimento (P\&D), menor é a importância do grau de escolaridade nos processos produtivos, mais baixo é o conteúdo tecnológico dos produtos e processos e menos relevante é a montagem dos produtos.

PALAVRAS-CHAVE Imitação entre empresas, mensuração da imitação, competências organizacionais, regime tecnológico, pesquisa e desenvolvimento.

Walter Bataglia batagliaw@gmail.com

Professor do Programa de Pós-Graduação em Administração de Empresas, Universidade Presbiteriana Mackenzie - São Paulo - SP, Brasil

Adilson Aderito da Silva adilson.asilva@gmail.com

Professor do Centro de Ciências Sociais e Aplicadas, Universidade Presbiteriana Mackenzie - São Paulo - SP, Brasil

Claudia Fernanda Franceschi Klement claudia.klement@gmail.com

Professora do Centro de Ciências Sociais e Aplicadas, Universidade Presbiteriana Mackenzie - São Paulo - SP, Brasil

Recebido em 01.04.2010. Aprovado em 15.02.2011

Avaliado pelo sistema double blind review

Editor Científico: Bento Alves da Costa Filho

\begin{abstract}
The objective of this paper was to identify the dimensions of the imitation construct between companies and to propose an operating procedure to measure it in business sectors. The imitation was conceptualized as a copy of capabilities. Putting them into operation was made possible by the ease of understanding them. The perceptions of 112 managers from 14 sectors of the Brazilian economy in the State of Sao Paulo were collected. The database of the Federation of Industries of the State of São Paulo (Fiesp) was used. The dimensions underlying the construct were identified by exploratory factorial analysis. The results indicate that the less that research and development (RED) activities are carried out, the less the importance of the level of schooling in productive processes, the lower the technological content of products and processes and the less significant the assembly of products, the greater the imitation tends to be in one sector.
\end{abstract}

KEYWORDS Imitation among enterprises, measurement of the imitation, organizational competences, technological regime, research and development.

RESUMEN El objetivo de este trabajo fue identificar las dimensiones del constructo imitación entre empresas y proponer un procedimiento operacional para su mensuración en los sectores empresariales. La imitación fue conceptuada como copia de competencias. Su operacionalización fue hecha por la facilidad de las comprender. Recogiéronse las percepciones de 112 gestores de 14 sectores de la economía brasileña en el estado de São Paulo (Fiesp). Las dimensiones subyacentes al constructo fueran identificadas por el análisis factorial exploratorio. Los resultados indican que la imitación en un sector tiende a ser mayor cuanto menos se realizan actividades de investigación y desarrollo (I $+D)$, menor es la importancia del grado de escolaridad en los procesos productivos, menor es el contenido tecnológico de los productos y procesos y menos relevante es el montaje de los productos.

PALABRAS CLAVE Imitación entre las empresas, mensuración de la imitación, competencias organizacionales, régimen tecnológico, investigación y desarrollo. 


\section{INTRODUÇÃO}

A dinâmica do ambiente competitivo cria mudanças externas que geram novas demandas, às quais as organizações precisam se adaptar. As firmas buscam aumentar sua habilidade de sobrevivência e a eficiência na obtenção de seus objetivos com base em de variações em suas rotinas e competências no sentido de um melhor alinhamento às novas demandas (BATAGLIA e MEIRELLES, 2009). As variações que levam a um melhor ajuste são selecionadas para ser retidas, duplicadas e propagadas internamente à firma em suas unidades. Uma das fontes dessas variações é a imitação (MINER e RAGHAVAN, 1999; KIM e RHEE, 2009), pois, ao observar além das fronteiras da firma, consegue-se capturar e absorver novos conhecimentos. A estratégia imitativa pode levar ao crescimento da firma (LIEBERMAN e ASABA, 2006), gerar vantagens que vão desde o acesso à competição com firmas inovadoras à entrada em novos mercados e também à obtenção de vantagens de custo de mão de obra. Enquanto empresas líderes tendem a se concentrar em inovações visando a redução de custos, as seguidoras baseiam-se mais em imitações, e sua entrada no mercado dificilmente pode ser evitada. $O$ imitador não necessita amortizar os pesados investimentos em P\&D e pode se beneficiar da experiência de outras empresas do mercado em que atua ou de outros mercados (ENKEL e GASSMANN, 2010; ESTIVALETE, PEDROZO, CRUZ, 2008; KIM e MINER, 2007). A imitação também pode levar as organizações à inovação. As empresas asiáticas começaram imitando as inovações ocidentais e conseguiram estabelecer um parque industrial competitivo e inovador (YAMAMURA, SONOBI, OTSUKA, 2005).

Esse é o contexto em que a presente pesquisa se desenvolveu, procurando oferecer uma contribuição à área com base em seu objetivo de identificar as dimensões do construto imitação entre empresas e propor um procedimento para sua mensuração. Assim, a questão central que este trabalho buscou responder foi: quais são as dimensões subjacentes ao construto imitação entre empresas? A resposta a essa questão gera ganhos tanto para a pesquisa na área de Administração Estratégica quanto para os praticantes da estratégia. Do ponto de vista da pesquisa, amplia as possibilidades de desenho e permite estabelecer as bases para trabalhos empíricos futuros que possam gerar maior conhecimento sobre a imitação e o seu papel no processo competitivo. Também torna possível que, baseados no controle da variável imitação, os pesquisadores escolham setores de interesse mais relevantes para suas questões de pesquisas. Do ponto de vista do praticante da estratégia, gera a possibilidade de identificar perfis ambientais.

Como principal resultado da pesquisa, obteve-se uma escala com 28 indicadores para mensuração do construto imitação entre empresas. Para apresentar a pesquisa, este artigo está estruturado em outras quatro seções. Na segunda, terceira e quarta seções, desenvolve-se uma breve revisão da literatura sobre a imitação, o ambiente tecnológico e o papel do conhecimento que serve de base teórica para este trabalho. A seguir, são apresentados os procedimentos metodológicos. Após, os resultados da análise de dados são apresentados e discutidos. Por fim, a seção de conclusões reúne as principais implicações da pesquisa, discute limitações e sugestões para estudos futuros.

\section{COMPETÊNCIAS ORGANIZACIONAIS, APRENDIZAGEM E IMITAÇÃO}

Na definição de Nelson e Winter (1982) e Dosi e Teece (1993), as firmas configuram-se como organizações com competências idiossincráticas de caráter tácito e cumulativo, as quais são organizadas por rotinas que guiam o processo de tomada de decisões nos âmbitos operacional e de investimento. As rotinas são padrões de interação que representam soluções para problemas particulares, agrupando ativos complementares e habilidades individuais. Portanto, nas rotinas é que reside o conhecimento gerado pelas atividades de aprendizado. Em outros termos, os processos de aprendizagem referem-se ao desenvolvimento das rotinas e competências das firmas. Eles são "locais", cumulativos e explicam, em grande parte, suas especificidades. A trajetória da firma e a existência de ativos complementares delimitam e, ao mesmo tempo, restringem os processos de aprendizado, e, por conseguinte, a definição de novas competências (Teece, 1986). São três as principais características dos processos de aprendizagem (DOSI e TEECE, 1993): i) envolvem habilidades organizacionais e individuais; ii) são fenômenos intrinsecamente sociais e coletivos; iii) ocorrem não só pela imitação entre indivíduos mas também pela participação conjunta de empresas na compreensão de problemas complexos. Nesse sentido, os processos de aprendizado podem se dar tanto interna quanto externamente à firma.

Para Cassiolato (2004), o aprendizado interno à organização ocorre a partir da ação pelo desenvolvimento de recursos humanos (educação formal, qualificação, treinamento, políticas de mão de obra) e mecanismos informais (circulação do conhecimento, efeito spill-over) e formais (atividades de P\&D) de aprendizado, e o aprendizado 
externo dá-se por imitação, pela interação com clientes e fornecedores dentro da cadeia produtiva, pela mobilidade de funcionários entre organizações e pela cooperação e por alianças com outras empresas. $\mathrm{O}$ aprendizado externo, em particular, necessita da existência de ampla capacitação interna para a realização da engenharia reversa. Assim, o aprendizado interno via atividade de pesquisa é condição necessária para o aprendizado externo. Por sua vez, o desempenho das atividades de P\&D depende do acesso da firma ao conhecimento externo (FEY e BIRKINSHAW, 2005), formando um ciclo virtuoso. Torres e Guimarães (2008) explicam que os processos de codificação e socialização dos conhecimentos adquiridos pelo aprendizado externo são os responsáveis pelas variações em rotinas, competências e estruturas existentes.

Para Haunschild e Miner (1997), o aprendizado externo dá-se por dois processos: 1) aprendizagem mimética, na qual a seleção do que será copiado é feita com base na experiência de outras organizações; e 2) aprendizagem pelo contato, que envolve a transmissão de rotinas por meio de relações formais e informais entre os membros das organizações. Essas autoras identificaram três bases para a escolha da rotina que será imitada na aprendizagem mimética: frequência, na qual se tende a adotar rotinas que são praticadas por um amplo número de organizações; legitimidade, na qual seletivamente se adotam práticas das organizações líderes; e resultados, na qual se copiam práticas adotadas com sucesso por outras empresas. Balestrin, Vargas e Fayard (2005) e Cunha e Melo (2006), por sua vez, destacam o papel da confiança e das relações informais nas cooperações interorganizacionais.

Lieberman e Asaba (2006) sugeriram a existência de duas abordagens para a imitação vinculada à aprendizagem mimética tendo como princípio fatores econômicos, tecnológicos, socioinstitucionais e afins à rivalidade competitiva. A primeira, a imitação baseada na rivalidade, tem seu fundamento na busca da contenção da rivalidade pelos competidores. A segunda, a imitação com base na informação, é caracterizada pela consideração dos gestores às informações implícitas nas ações dos demais atores ambientais. Essas abordagens são complementares (DELIOS, GAUR, MAKINO, 2008).

A imitação baseada na rivalidade ocorre quando as firmas competidoras possuem o mesmo porte, a mesma capacidade financeira e o mesmo posicionamento de mercado, apresentando intensa competição entre si. Nessa situação, os competidores usualmente buscam manter sua posição sem se enveredar por hostilidades destrutivas que possam corroer os preços e a lucratividade. Para tanto, adotam estratégias de homogeneização, que consistem em seguir o comportamento dos rivais visando conter a rivalidade. O conhecimento de que o rival responderá com ações similares diminui o incentivo para a busca agressiva da vantagem competitiva, preservando-se assim o status entre os competidores.

A imitação baseada na informação usualmente é adotada por empresas seguidoras em condições assimétricas de posição de mercado e acesso a recursos em relação aos líderes ou por empresas líderes em situações de incerteza, visando reduzir os riscos envolvidos na tomada de decisões. Trata-se, respectivamente, da tentativa de legitimar as escolhas realizadas buscando prestígio pessoal e/ou organizacional, e/ou imitar uma opção qualitativamente superior (BARRETO e BADEN-FULLER, 2006; DOBREV, 2007; RACHID, BRESCIANI FILHO, GITAHY, 2001; STABER, 2010).

A organização busca, por meio da aprendizagem, desenvolver suas rotinas e adquirir outras, focando o "aprender fazendo" e o "aprender com os outros". A aprendizagem de rotinas e competências entre organizações tem sido denominada, na literatura, de aprendizagem interorganizacional (INGRAM, 2005). A aprendizagem com base na própria experiência pode ser a fonte da produção eficiente e da sustentação da vantagem competitiva, pois, ao aperfeiçoar uma rotina, é provável que os custos de produção diminuam. Porém, esse tipo de aprendizagem pode levar a uma armadilha, se as rotinas existentes se tornarem desajustadas às demandas do ambiente competitivo a partir de variações externas (LEVINTHAL e MARCH, 1993; JANSEN, VAN DEN BOSCH, VOLBERDA, 2006; WANG e LI, 2008). Nesse sentido, a organização deve dividir sua atenção e seus recursos, balanceando suas atividades de prospecção de novas rotinas e exploração das rotinas existentes (MARCH, 1991; KIM e RHEE, 2009). A prospecção corresponde à geração e experimentação de maneiras alternativas de fazer as coisas e a exploração refere-se à aprendizagem obtida por meio do processo de otimizar as rotinas existentes. Quando ocorre muita prospecção, usualmente, os custos de experimentação aumentam sem retorno no curto prazo. E quando aumenta o nível de exploração, simultaneamente, melhora o desempenho no curto prazo e se elevam os riscos de mortalidade no longo prazo.

Enfim, há uma forte associação entre inovação, aprendizagem e imitação. Assim, os diferentes processos de aprendizagem são fundamentais na transmissão, comercialização e aperfeiçoamento das inovações, bem como na apropriação dos ganhos resultantes do processo inovativo. Por outro lado, quanto maiores forem as barreiras de aces- 
so ao conhecimento relevante em um setor, maior será a dificuldade para a imitação. Essas barreiras são definidas, sobretudo, pelo regime tecnológico, apresentado a seguir.

\section{REGIME TECNOLÓGICO E ESTRATÉGIA DE IMITAÇÃO}

O regime tecnológico é definido levando-se em conta as condições de oportunidade, cumulatividade, apropriabilidade e natureza da base de conhecimento vigentes em um dado setor de atividade empresarial (MALERBA e ORSENIGO, 1993). A cumulatividade está relacionada à ideia de que as inovações de hoje e as atividades de $\mathrm{P} \& \mathrm{D}$ são alicerces para as inovações de amanhã. A oportunidade tecnológica reflete a facilidade da atividade inovativa. Essas possibilidades podem ser avaliadas tanto em relação ao volume de oportunidades (depende do setor e da tecnologia em questão) quanto ao grau de granularidade (pervasiveness) (amplitude da aplicabilidade de novos conhecimentos em produtos, processos e mercados). As condições de apropriabilidade referem-se às possibilidades de proteção das inovações de imitações. Por fim, a base de conhecimento é definida analisando-se duas dimensões: grau de conhecimento tácito (tacitness) e complexidade. O grau de conhecimento tácito (tacitness) é tanto menor quanto mais articulado e de fácil acesso for o conheci- mento. O grau de complexidade é definido pelo grau de interconexão de diferentes conhecimentos/disciplinas, bem como pela própria variedade de competências organizacionais necessárias nos principais processos da firma

As condições do regime tecnológico mais favoráveis para as estratégias de imitação são a alta oportunidade, a baixa apropriabilidade e a alta cumulatividade (Quadro 1). A cumulatividade favorece as estratégias de imitação em função de estarem associadas à inovação incremental.

Três conceitos são fundamentais na análise do processo de imitação (TEECE, 1986): regime de apropriabilidade, ativos complementares e paradigma dominante (design dominante). O regime de apropriabilidade é definido por duas dimensões: instrumentos legais (patentes, direitos autorais, segredos industriais) e natureza da tecnologia do produto e do processo produtivo (grau de conhecimento tácito e complexidade). A defesa da imitação dá-se com base em estratégias que visam aumentar o grau de apropriabilidade da inovação lançada no mercado. Por outro lado, o sucesso na comercialização de uma inovação depende da junção do novo conhecimento com ativos ou capacidades complementares, tais como infraestrutura de máquinas e equipamentos ou mão de obra qualificada, que permitam a produção competitiva da inovação, serviços de marketing e suporte pós-venda.

O estágio de evolução do design dominante também contribui no potencial de imitação. Normalmente, quando

Quadro 1 - Estratégias tecnológicas básicas

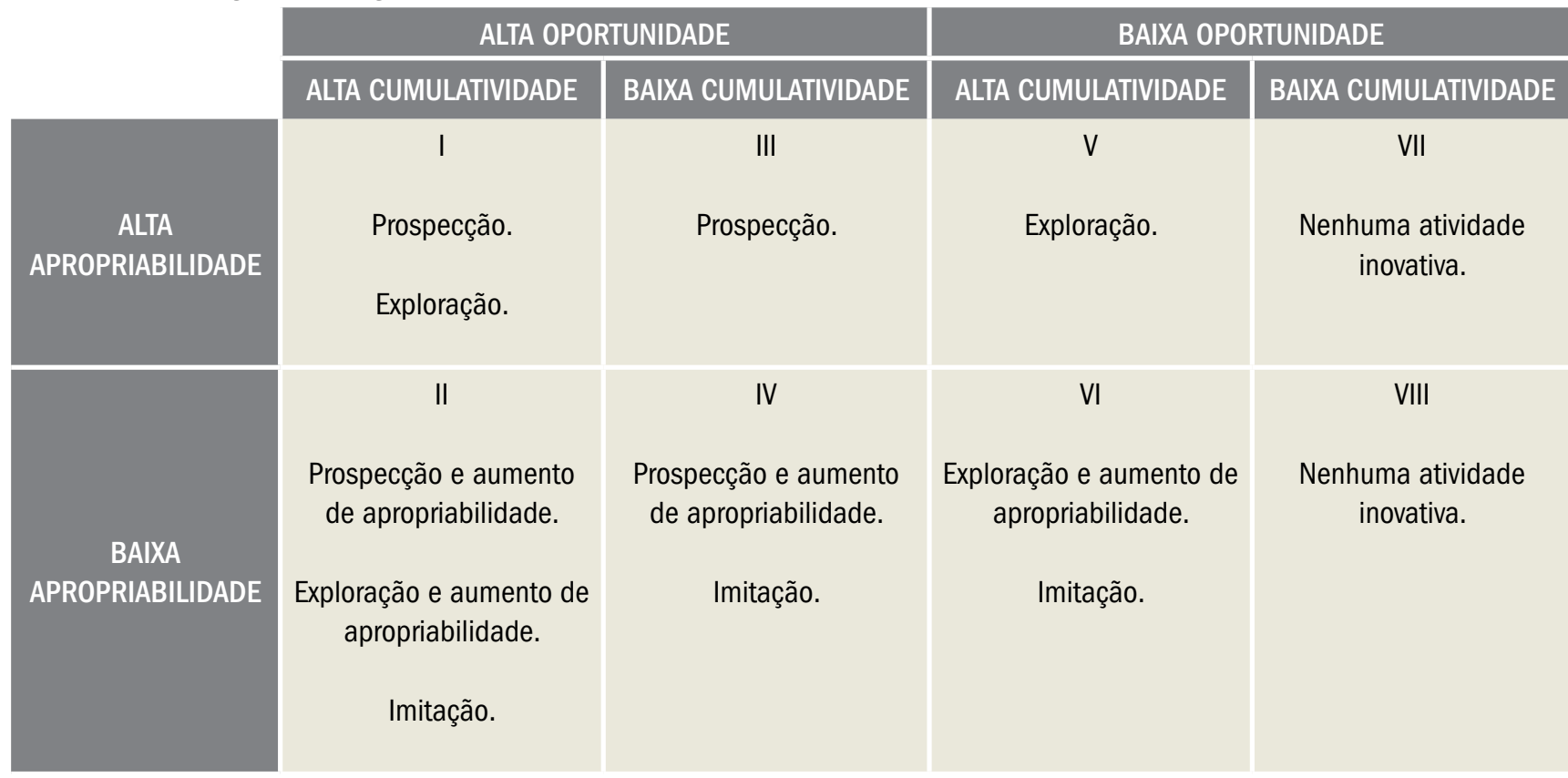

Fonte: MALERBA e ORSENIGO, 1993, p. 61 (tradução nossa). 
um produto é lançado, os imitadores entram no mercado, promovendo mudanças significativas ou simplesmente copiando. Nesse processo, em muitos casos, o design dominante acaba sendo o do imitador e não o do inovador. A necessidade de ativos complementares na fase de difusão do design dominante aumenta a probabilidade de imitação. Sobretudo quando os ativos são especializados e altamente dedicados. Nesse caso, as firmas estabelecidas (fornecedoras ou possuidoras desses ativos) têm maior vantagem diante dos inovadores, tendo em vista que maiores serão os riscos envolvidos na aquisição desses ativos via contratos. De acordo com Teece (1986) (Figura 1), a opção pela integração vertical facilita o alinhamento de incentivos e permite um maior controle desse processo. Por outro lado, a opção pela aquisição dos ativos complementares via contratos é a melhor estratégia quando o regime de apropriação é forte e a oferta desses ativos é competitiva. Além disso, acordos contratuais com empresas fortes (de marca reconhecida no mercado) dão credibilidade à inovação, principalmente quando a empresa inovadora é desconhecida no mercado. Contudo, o tempo de aquisição dos ativos complementares e a necessidade de caixa são fatores que podem desestimular a integração vertical, mesmo quando o regime de apropriação é fraco.

Em resumo, barreiras de acesso ao conhecimento relevante em um setor definem um regime de apropriabilidade forte e dificultam a imitação. Por outro lado, um regime de baixa apropriação combinado com a necessidade de

Figura 1 - Diagrama correspondente à decisão de integração versus contrato

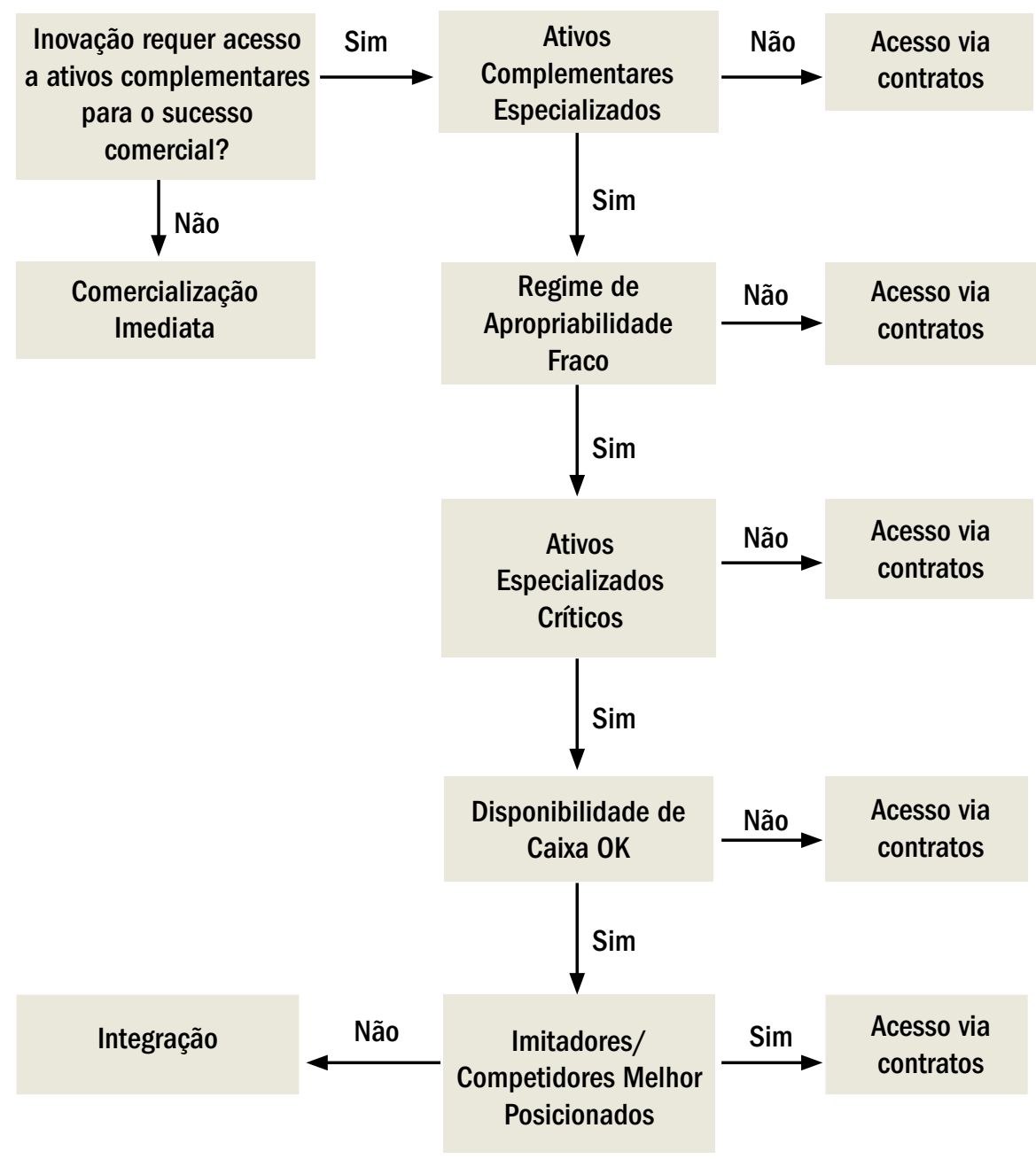

Fonte: TEECE, 1986, p. 296 (tradução nossa). 
ativos complementares especializados é um fator que contribui para a imitação.

\section{CONHECIMENTO E REPLICACÃO DE COMPETÊNCIAS NA IMITAÇÃOO}

A base da imitação de uma inovação é a capacidade de se replicarem as competências e rotinas de produção e venda de um novo produto ou serviço sem o consentimento da firma que lançou a inovação no mercado. A transferência e comunicação das novas habilidades e competências para a firma imitadora ocorrem com base na mobilização das estruturas relacionais e dos esquemas de codificação compartilhados pelos grupos sociais vinculados às rotinas existentes (KOGUT e ZANDER, 1992; TORRES e GUIMARÃES, 2008). Nesse sentido, Rogers (1980, 2003) aponta atributos de uma inovação que influenciam a velocidade de sua difusão: a vantagem relativa (relative advantage) percebida na sua adoção; a compatibilidade (compatibility) com os valores e necessidades do adotante; a complexidade (complexity) no que se refere à dificuldade de sua compreensão; a sua experimentabilidade (trialability) ou possibilidade de se experimentá-la antes da adoção; e sua observabilidade (observability) ou possibilidade de visualização antecipada do resultado de sua adoção.

Winter (1987) propôs uma taxonomia para a facilidade de replicação de uma inovação com base no conhecimento utilizado nas competências organizacionais associadas à sua implementação (Quadro 2). Uma posição mais à direita no continuum de cada dimensão indica maior facilidade de replicação. A dimensão tácito/articulável trata do quanto o conhecimento é articulável, facilitando sua comunicação. Essa dimensão possui duas subdimensões. A subdimensão ensinável/não ensinável, que se refere à possibilidade de um conhecimento tácito ser ensinado via interação social, e a subdimensão articulado/não articulado, que distingue conhecimentos que já estão articulados dos que não estão. A dimensão observável/não observável refere-se à possibilidade de que o conhecimento seja percebido e apreendido via observação. A dimensão complexo/ simples trata do grau de complexidade do conhecimento em função das diferentes áreas e disciplinas envolvidas. Por fim, a dimensão dependente/independente refere-se a quanto o conhecimento está associado à interação entre diferentes etapas ou áreas da organização.

Zander e Kogut (1995), com base nos trabalhos de Rogers (1980) e Winter (1987), propuseram cinco dimensões para caracterização da facilidade de comunicação e compreensão do conhecimento relevante existente nas competências organizacionais (ver também Kogut e Zander, 1993). Essas dimensões foram elaboradas por meio de um estudo de caso múltiplo no setor de manufatura, envolvendo oito inovações e três empresas. São elas: 1) codificabilidade (codifiability) ou grau de facilidade de codificar um conhecimento; 2) ensinabilidade (teachability) ou grau de facilidade para ensinar um conhecimento; 3) complexidade (complexity) ou grau de dificuldade de um conhecimento relativamente à composição de diversas áreas tecnológicas e/ou científicas; 4) observabilidade (product observability) ou grau de dificuldade de um concorrente em copiar um produto baseado em sua observação; e 5) dependência sistêmica (system dependence), ou seja, o grau de dependência de um conhecimento de outros sistemas.

Observe-se que essas dimensões estão associadas à apropriabilidade e à natureza da base de conhecimento vigentes no regime tecnológico dos setores. No entanto, conforme apresentado na seção anterior, a teoria indica que a imitação entre empresas também está associada a outras características do regime tecnológico, como cumulatividade e oportunidade. Configura-se, dessa forma, um problema de validade de conteúdo na escala proposta. De qualquer forma, esses autores operacionalizaram essas cinco dimensões, gerando um procedimento para mensuração da imitação entre empresas com base na percepção

\section{Quadro 2 - Taxonomia do conhecimento organizacional}

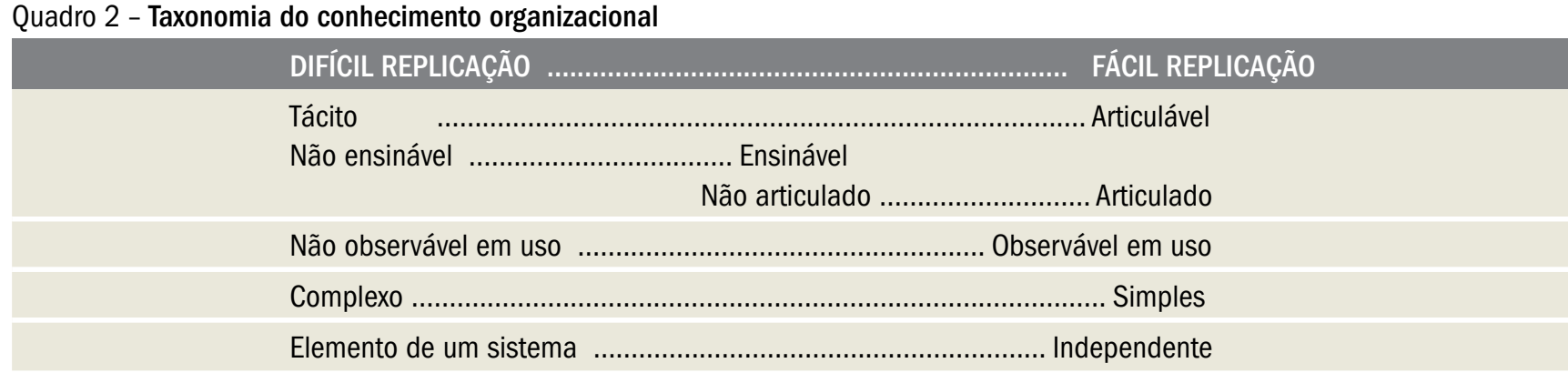

Fonte: Adaptado de WINTER, 1987, p. 170 (tradução nossa). 
dos gestores, que foi aplicado em 35 respondentes. A escala foi ajustada pelo grau de confiabilidade determinado pelo Alfa de Cronbach para cada dimensão.

\section{MÉTODO}

O propósito deste trabalho foi dar continuidade à discussão sobre a criação e a validação de um procedimento de mensuração do construto imitação entre as empresas. Partiu-se do trabalho de Zander e Kogut (1995). Em função do problema de validade de conteúdo da escala proposta por esses autores, apresentado na seção anterior, partiu-se do objetivo de identificação exploratória das dimensões componentes do construto imitação entre empresas. Para tanto, desenvolveu-se uma revisão da literatura, investigando-se as características do regime tecnológico relacionadas à imitação. As características foram operacionalizadas com base em indicadores, para os quais foram colhidos dados primários nos setores de atividade empresarial. A técnica estatística utilizada para extrair exploratoriamente as dimensões dos dados foi a Análise Fatorial Exploratória (AFE). Também se testou, via técnica estatística de análise de variância (Anova), a existência de diferenças entre os escores das dimensões extraídas em função dos setores de atuação e do porte das empresas.

O universo da pesquisa foi definido pelo conjunto de empresas da indústria de transformação da Classificação Nacional das Atividades Empresariais (CNAE) (2004) constantes na base da Fiesp. O setor de manufatura foi escolhido para que os resultados pudessem ser comparados com as pesquisas anteriores. O estudo de campo limitou-se às empresas do Estado de São Paulo pela facilidade de acesso dos pesquisadores à região geográfica. Para tal, foi selecionada por conveniência, da base da FIESP, uma amostra com 3.000 empresas distribuídas proporcionalmente por setor, às quais foram enviadas mensagens eletrônicas explicitando os objetivos da pesquisa e uma carta-convite. Dessas mensagens, 241 retornaram com endereços inválidos e as 2.759 restantes, consideradas potenciais participantes, foram contatadas por telefone. A unidade de análise foram gestores até dois níveis hierárquicos abaixo do executivo principal, visando evitar vieses de interpretação do ambiente competitivo causados pela influência da área funcional.

O instrumento de coleta foi elaborado com assertivas referentes às dimensões identificadas na revisão bibliográfica sobre imitação entre empresas propostas por Freeman e Soete (1997), Teece (1986), Malerba e Orsenigo (1993) e Zander e Kogut (1995), resultando num questionário de autopreenchimento com escala de concordância tipo Likert, variando de 1 (discordo totalmente) a 5 (concordo totalmente), exceto as duas variáveis relativas ao registro de patentes elaboradas em escala binária. Foram analisados os seguintes aspectos da imitação, agrupados em quatro dimensões centrais: 1) apropriabilidade, que inclui os aspectos de patentes, desenvolvimento contínuo de novos processos e produtos, ensinabilidade, observabilidade, codificabilidade e dependência sistêmica; 2) complexidade dos processos, na qual se incluem o nível de escolaridade necessário aos funcionários, os diferentes tipos de processos de manufatura envolvidos e a capilaridade do conhecimento tecnológico utilizado pela firma; 3) cumulatividade, relacionada ao lançamento de novos produtos, processos de manufatura, modelos organizacionais e benefícios radicalmente distintos dos existentes; e 4) oportunidade, na qual se incluem a intensidade de $\mathrm{P} \& \mathrm{D}$ e a difusão de conhecimento interna à firma. O total foi de 43 assertivas e quatro questões caracterizadoras do perfil da empresa e do respondente.

\section{RESULTADOS E ANÁLISE}

A amostra resultante dos contatos por endereço eletrônico e por telefone foi de 112 respondentes, distribuídos em 14 setores da indústria de transformação brasileira classificados no nível de dois dígitos da CNAE (Tabela 1).

O tempo médio de atuação dos gestores respondentes no setor é de aproximadamente 12,1 anos com desvio padrão 8,6 anos, e o tempo médio na empresa atual é de 8,9 anos com desvio padrão 7,5 anos. As empresas foram classificadas por porte de acordo com as faixas propostas pelo Serviço Brasileiro de Apoio às Micro e Pequenas Empresas (Sebrae), considerando as faixas do número de empregados (Tabela 2).

Como nesta pesquisa buscou-se também testar diferenças entre os escores produzidos em cada dimensão identificada do construto imitação, segundo o setor de atuação e o porte das empresas pesquisadas, o passo seguinte foi determinar o tamanho do efeito $\left(f^{2}\right)$ a ser detectado com base na significância $(\alpha)$, no tamanho da amostra e no poder estatístico (1- $\beta$ ), com o objetivo de garantir a consistência e maior precisão dos testes. Para se detectarem diferenças entre agrupamentos com características distintas por meio do teste Anova, Cohen (1988) definiu três níveis de efeito do tamanho da amostra: 0,10 (baixo); 0,30 (médio) e 0,40 (elevado). Nesse estudo, foi estipulado o nível de significância $(\mathrm{a}=0,10)$ e poder estatístico $(1-\beta$ 
$=0,80)$, o que resultou no tamanho do efeito $\left(f^{2}=0,30\right)$ para a amostra de 112 empresas.

Realizou-se um pré-teste com as 30 primeiras empresas respondentes, para se testarem as consistências internas e a confiabilidade dos indicadores propostos em cada construto por meio do Alfa de Cronbach (NUNNALLY e BERNSTEIN, 1994). O pré-teste apresentou índices mínimos de confiabilidade para a maioria dos construtos. Efetuados ajustes semânticos para melhoria do questionário, procedeu-se à finalização da pesquisa de campo. As empresas do pré-teste foram mantidas na amostra final. $O$ valor da estatística alfa para a amostra final indicou baixa confiabilidade para os construtos ensinabilidade e dependência sistêmica, abaixo do patamar mínimo de 0,60 sugerido por Nunnally e Bernstein (1994), conforme pode ser observado na Tabela 3.
Após a finalização do levantamento dos dados, deu-se início ao seu processamento pela avaliação dos resultados produzidos pelo software Statistical Package for the Social Sciences (SPSS) para as técnicas de estatística descritiva, com o objetivo de identificar valores extremos, ausência de dados nas respostas e também para se testar a normalidade das distribuições das variáveis. As significâncias estatísticas do teste de normalidade de Kolmogorov-Smirnov demonstraram que o pressuposto de normalidade univariada dos indicadores não foi violado. Iniciou-se, então, o estudo exploratório dos dados com o processamento da análise fatorial pelo método de componentes principais com rotação Varimax que se mostrou adequado pelo indice da amostra Kaiser-Meyer-Olkin $(\mathrm{KMO}=0,627) \mathrm{e}$ pela estatística Qui-Quadrado para o teste de Bartlett $\left(\chi^{2}\right.$ $=1186,32$; g.l $=378$ e sig. $=0,000)$. Esse primeiro proces-

Tabela 1 - Distribuição dos respondentes por setor de atuação

\begin{tabular}{|c|l|r|r|}
\hline CNAE & \multicolumn{1}{|c|}{ SETOR } & AMOSTRA & FREQ. (\%) \\
\hline 15 & Fabricação de Produtos Alimentícios e Bebidas & 6 & 5,40 \\
\hline 18 & Confecção de Artigos do Vestuário e Acessórios & 3 & 2,70 \\
\hline 19 & Fabricação de Calçados & 2 & 1,80 \\
\hline 22 & Edição, Impressão e Gravação & 25 & 22,30 \\
\hline 24 & Fabricação de Produtos Químicos & 5 & 4,40 \\
\hline 26 & Fabricação de Artigos de Borracha e Plástico & 11 & 9,80 \\
\hline 27 & Fabricação de Produtos de Minerais Não Metálicos & 2 & 1,80 \\
\hline 28 & Fabricação de Produtos Diversos de Metal & 3 & 2,70 \\
\hline 31 & Fabricação Equip. p/ Distrib. Contr. Energia Elétr. & 6 & 5,40 \\
\hline 36 & Fabricação Peças e Acess. p/ Veíc. Automotores & 9 & 8,00 \\
\hline 45 & Fabricação de Produtos Diversos & 14 & 12,50 \\
\hline 72 & Construção & 18 & 16,00 \\
\hline TOTAL & Serviços de Informática & 3 & 2,70 \\
\hline
\end{tabular}

Tabela 2 - Classificação dos respondentes por tamanho das empresas

\begin{tabular}{|l|c|c|c|c|}
\hline & FREQUÊNCIA & PERCENTUAL & $\begin{array}{c}\text { PERCENTUAL } \\
\text { VÁLIDO }\end{array}$ & $\begin{array}{c}\text { PERCETUAL } \\
\text { ACUMULADO }\end{array}$ \\
\hline Acima de 500 & 21 & 18,80 & 18,80 & 18,80 \\
\hline De 100 a 499 & 35 & 31,30 & 31,30 & 50,00 \\
\hline Menos que 99 & 56 & 50,00 & 50,00 & 100,00 \\
\hline TOTAL & 112 & 100,00 & 100,00 & \\
\hline
\end{tabular}


samento resultou na extração de 10 fatores pelo critério do eigenvalue maior que 1 e apresentou uma variância explicada de $76,6 \%$.

No primeiro e no quarto fatores, ficaram agrupadas, respectivamente, as quatro assertivas relacionadas aos tipos de processos e as três assertivas de nível de escolaridade. Esses dois fatores representam as subdimensões propostas para complexidade do conhecimento. No segundo, ficaram carregadas quatro das cinco assertivas elaboradas para captar a intensidade de pesquisa e desenvolvimento; no sétimo e oitavo fatores, ficaram carregadas as cinco assertivas de capilaridade de conhecimento. Esses três fatores representam as duas subdimensões propostas para oportunidade de mercado. No terceiro, quinto, nono e décimo fatores, ficaram respectivamente carregadas três das quatro assertivas de observabilidade; três das sete assertivas de codificabilidade ou conhecimento tácito; as duas assertivas relacionadas ao registro de patentes e as duas assertivas propostas para desenvolvimento contínuo. Esses quatro fatores representam as subdimensões propostas para apropriabilidade. No sexto fator, ficaram carregadas duas das quatro assertivas de cumulatividade de conhecimento. O processamento da análise fatorial indicou oito variáveis com valores de comunalidade abaixo de 0,50 e, a exemplo das sete assertivas propostas inicialmente para os fatores dependência sistêmica e ensinabilidade, que apresentaram consistências internas abaixo do patamar 0,60, foram retiradas das análises subsequentes, conforme recomendado por Nunnally e Bernstein (1994). Portanto, das 43 assertivas inicialmente propostas no estudo, apenas 28 permaneceram para as análises finais.

A análise acima revelou alguns fatores que poderiam refletir um mesmo conceito, e também um pequeno acréscimo de apenas $11,88 \%$ na porção de variância explicada do sétimo ao décimo fator extraído. Como um dos objetivos da análise fatorial é a simplificação das informações, sem, contudo, perder a essência na explicação dos conceitos subjacentes (HAIR e outros, 2005), um segundo processamento dessa técnica multivariada foi efetuado pelo critério predefinido de extração dos fatores, ou seja, estipulando-se previamente o número de fatores ortogonais a extrair.

O novo processamento foi efetuado para extração de sete fatores ortogonais e produziu o índice de adequação de Kaiser-Meyer-Olkin $(\mathrm{KMO}=0,638)$, a estatística Qui-Quadrado aproximada para o teste de Bartlett $\left(\chi^{2}=\right.$ 1276,038; g.l $=378$ e sig. $=0,000)$ e a variância explicada de 63,053\%. Como pode ser observado na Tabela 4, no primeiro fator, agruparam-se as assertivas relativas às atividades de $\mathrm{P} \& \mathrm{D}$. Tal fator foi denominado apropriação de oportunidades. No segundo fator, ficaram carregadas duas assertivas que abrangem o grau de inovação do conteúdo tecnológico dos novos produtos e processos produtivos e três assertivas sobre a importância do grau de escolaridade dos funcionários. Tal fator foi denominado cumulatividade de conhecimento técnico. O terceiro fator caracterizou-se pelo agrupamento de assertivas relacionadas aos conhecimentos e disciplinas distintas envolvidos no processo produtivo. Foi denominado complexidade. $\mathrm{O}$

Tabela 3 - Alfa de Cronbach para amostra pré-teste e amostra final

\begin{tabular}{|l|l|l|l|}
\hline \multicolumn{1}{|c|}{ CONSTRUTO TEÓRICO } & ASSERTIVAS & ALFA PRÉ-TESTE & ALFA FINAL \\
\hline Apropriabilidade & & & \\
\hline Patentes & 2 & 0,750 & 0,772 \\
\hline Desenvolvimento contínuo & 2 & 0,766 & 0,683 \\
\hline Ensinabilidade & 3 & 0,499 & 0,382 \\
\hline Observabilidade & 4 & 0,729 & 0,757 \\
\hline Codificabilidade & 7 & 0,478 & 0,684 \\
\hline Dependência sistêmica & 4 & 0,443 & 0,509 \\
\hline Complexidade & & & \\
\hline Nível de escolaridade & 3 & 0,609 & 0,732 \\
\hline Tipos de processos & 4 & 0,684 & 0,820 \\
\hline Capilaridade conhecimento & 5 & 0,763 & 0,688 \\
\hline Oportunidade & 4 & 0,854 & 0,812 \\
\hline Cumulatividade & 5 & 0,884 & 0,788 \\
\hline
\end{tabular}


quarto foi definido como observabilidade, dado que nele ficaram agrupadas três das quatro assertivas propostas para essa subdimensão da apropriabilidade. No quinto fator, ficaram carregadas as cinco assertivas propostas para capilaridade do conhecimento necessário e as duas assertivas sobre a importância da melhoria contínua para proteção dos segredos industriais. Esse fator foi definido como dependência de conhecimento externo. O sexto fator foi interpretado por codificabilidade, em que ficaram carregadas três das sete assertivas propostas. Finalmente, no sétimo fator, ficaram carregadas as assertivas relacionadas com novas patentes, denominado apropriabilidade. Na Tabela 4, também podem ser encontrados os eigenvalues, a consistência interna e a variância explicada associados a cada fator. Todos os fatores apresentam confiabilidades adequadas com valores do Alfa de Cronbach acima de 0,60, conforme proposto por Nunnally e Bernstein (1994).
Tabela 4 - Matriz de fatores extraídos no segundo processamento

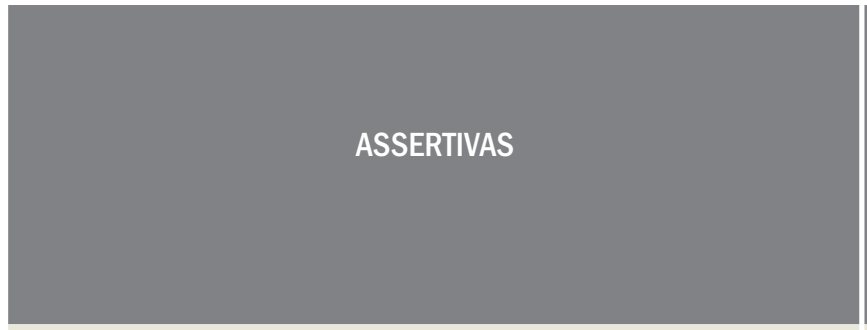

Nossa empresa não realizou pesquisa e desenvolvimento entre 2005-2007

Nossa empresa não utiliza difusão de conhecimento internamente (departamentos/unidades)

Nossa empresa não desenvolve produtos inovadores para 0 mercado

A empresa não possuía departamento específico de $P \& D$ entre 2005 e 2007

Os processos de manufatura dos novos produtos no nosso setor não incorporam tecnologias radicalmente diferentes das existentes

Não é necessário alto grau de escolaridade para o pessoal com ocupação semiqualificada

Novos produtos lançados no nosso setor não embutem tecnologias radicalmente diferentes

Não é necessário alto grau de escolaridade para o pessoal com ocupação auxiliar e básica

Não é necessário alto grau de escolaridade para o pessoal com ocupação qualificada

Processos para mudança da forma do material (ex. moldagem) não são importantes

Processos para montagem de diferentes partes do produto (ex. solda) não são importantes

Processos para dar aos materiais certas dimensões (ex. torno) não são importantes

Processos para mudança física do material (ex. polimento) não são importantes (continua)
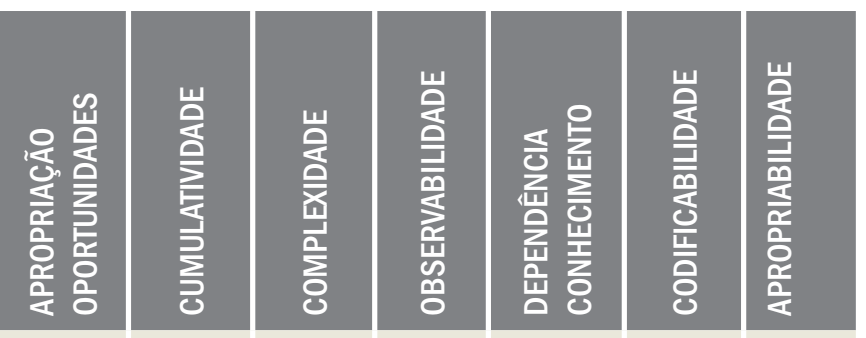

0,836

0,750

0,702

0,662

0,739

0,735

0,735

0,718

0,512

0,821

0,809

0,771

0,747 
Testando e utilizando nossos produtos, a concorrência pode facilmente aprender sobre como eles são produzidos

A concorrência pode facilmente aprender sobre como se produzem nossos produtos, analisando-os cuidadosamente

Analisando as descrições dos catálogos, a concorrência facilmente aprende sobre como se produzem nossos produtos

Nossa empresa é dependente de clientes e consumidores sobre conhecimentos necessários à produção e comércio de nossos produtos

Desenvolvimento contínuo é importante para nos proteger da imitação pela concorrência

Nossa empresa depende de universidades e institutos de pesquisa sobre os conhecimentos necessários à produção ou comércio Nossa empresa depende de consultorias e centros de capacitação sobre conhecimentos necessários à produção ou comércio

Modificações contínuas em produtos são importantes para nos proteger da imitação

A empresa é dependente dos fornecedores em relação aos conhecimentos necessários à produção e comercialização de nossos produtos

A empresa é dependente dos concorrentes em relação aos conhecimentos necessários à produção e comercialização de nossos produtos

Grande parte do controle da produção está embutida em software padrão parametrizável

Grande parte do controle dos processos da empresa (exceto manufatura) está embutida em software padrão parametrizável (ex. SAP)

0 conhecimento aprendido sobre novos produtos e seu processo produtivo é facilmente transformado em cursos e manuais

Nossa empresa (ou grupo) não registrou patentes no exterior para proteger inovações desenvolvidas de 2005 a 2007

Nossa empresa (ou grupo) não registrou patentes no Brasil para proteger inovações desenvolvidas de 2005 a 2007

Autovalores

Consistência Interna (Alfa de Cronbach)

Variância Explicada (\%)

Observa-se que as dimensões complexidade e observabilidade propostas por Winter (1987) e Zander e Kogut (1995) tiveram suas significâncias confirmadas. A dimensão codificabilidade, presente no trabalho de Zander e Kogut (1995) com o mesmo nome e no trabalho de Winter (1987) com o nome tácito/articulável, também foi confirmada. As dimensões ensinabilidade

\begin{tabular}{|c|c|c|c|c|c|c|}
\hline & & & 0,791 & & & \\
\hline & & & 0,774 & & & \\
\hline & & & 0,717 & & & \\
\hline & & & & 0,617 & & \\
\hline & & & & 0,604 & & \\
\hline & & & & 0,589 & & \\
\hline & & & & 0,536 & & \\
\hline & & & & 0,502 & & \\
\hline & & & & 0,476 & & \\
\hline & & & & 0,441 & & \\
\hline & & & & & 0,751 & \\
\hline & & & & & 0,689 & \\
\hline & & & & & 0,604 & \\
\hline & & & & & & 0,838 \\
\hline & & & & & & 0,785 \\
\hline 4,534 & 3,036 & 2,545 & 2,204 & 1,980 & 1,875 & 1,480 \\
\hline 0,813 & 0,769 & 0,821 & 0,791 & 0,656 & 0,664 & 0,773 \\
\hline 10,605 & 9,844 & 9,818 & 9,195 & 8,543 & 7,941 & 7,107 \\
\hline
\end{tabular}

e dependência sistêmica, também propostas por esses autores, não apresentaram significância. Esses resultados refletem uma evolução, já que o trabalho de Winter (1987) é teórico e Zander e Kogut (1995) não testaram a unidimensionalidade das dimensões internas e a validade convergente do construto imitação que propuseram. Vale destacar que os resultados desta 
pesquisa apontaram quatro novas dimensões da imitação em relação aos trabalhos anteriores: oportunidade, cumulatividade, dependência de conhecimento externo e apropriabilidade.

Ainda se avançando em relação aos trabalhos anteriores, observa-se que a utilização da Análise das Componentes Principais (ACP) permite gerar escores individuais das empresas em cada uma das dimensões (fatores) obtidas pela combinação linear dos escores padronizados de suas variáveis. Os escores das empresas distribuem-se segundo uma normal com média zero e variância unitária. Logo, torna-se possível situar a posição relativa das empresas para cada uma das dimensões da imitação, possibilitando a criação de um índice de imitação. Assim, um respondente que apresenta um escore elevado no fator "observabilidade", por exemplo, o qual está relacionado com a facilidade com que o concorrente aprende a produzir seu produto, indicará parcialmente o grau em que a sua empresa está suscetível à imitação pelos concorrentes.

Nesse sentido, é importante observar que, na ACP, a combinação linear de variáveis que reflete a maior variância possível do banco de dados é alocada no primeiro fator, e assim, sucessivamente, até o último fator extraído. Consequentemente, uma relação hierárquica é estabelecida entre as dimensões em termos de variância explicada, a qual pode ser avaliada segundo a magnitude do autovalor. Assim, na elaboração do índice, faz-se necessário ponderar o escore individual das empresas nos fatores pela variância explicada por meio do autovalor de cada fator. O índice de imitação foi definido pela seguinte equação:

$$
\operatorname{IMITAÇÃO}_{\mathrm{i}}=\sum_{\mathrm{j}} \lambda_{\mathrm{j}}^{*} \mathrm{~F}_{\mathrm{ji}} / \sum_{\mathrm{j}} \lambda_{\mathrm{j}}
$$

Sendo:

$$
\mathrm{F}_{\mathrm{ji}}=\sum_{\mathrm{n}} \mathrm{a}_{\mathrm{nj}}{ }^{*} z_{\mathrm{ni}}
$$

Em que:

$\mathrm{F}_{\mathrm{ji}}=$ Escore fatorial do fator $\mathrm{F}_{\mathrm{j}}$ para o gestor $\mathrm{i}$

$\lambda_{\mathrm{j}}=$ Eigenvalue do fator $\mathrm{F}_{\mathrm{j}}$

$z_{\mathrm{ni}}=$ Valor percebido pelo gestor i para o indicador $z_{n}$

$a_{n j}=$ Coeficiente do indicador $z_{n}$ no escore fatorial do

fator $F_{j}$

$\mathrm{i}=1, \ldots, 112$ (número de respondentes)

$\mathrm{j}=1, \ldots, 7$ (número de fatores)

$\mathrm{n}=1, \ldots, 23$ (número de indicadores)
Com base no índice proposto, foram calculados os escores de imitação percebidos para cada uma das empresas pesquisadas e, a seguir, buscou-se testar a existência de possíveis diferenças nos valores obtidos por conta do setor de atuação e do porte das empresas. Para tal, utilizou-se Anova, tomando como variável escalar (dependente) os escores do índice de imitação e como variáveis categóricas (prognosticadoras) o setor de atuação e o porte das empresas. O teste foi processado em três fases: na primeira, foram testadas as diferenças segundo o setor de atuação na segunda, por setor de atuação levando-se em consideração o porte das empresas (pequena, média e grande); e, finalmente, as diferenças nos escores de imitação foram testadas segundo o porte das empresas independentemente do setor de atuação.

A análise de variância requer a normalidade da variável dependente e que haja homogeneidade de variâncias dos grupos testados, ou seja, grupos de empresas classificadas por porte (pequenas, médias e grandes) e por setor. A avaliação da normalidade do índice de imitação com o teste de Kolmogorov-Smirnov (estatística $=0,047$, g.l $=$ 112 e sig. $=0,200$ ) indica a não violação do pressuposto de normalidade. O mesmo foi observado em relação ao teste de Levine para a homogeneidade da variância entre os grupos de empresas (Tabela 5).

Para testar as diferenças nos escores do índice de imitação com relação aos setores de atuação ( $1^{a}$ fase), foram escolhidos os seis setores com maior número de respondentes entre os 14 setores, ao nível de dois dígitos da CNAE $(15 ; 22 ; 25 ; 31 ; 34$ e 36) (Tabela 1). Essa escolha visou a atender o critério de quantidade mínima de respondentes por grupo pesquisado para utilização do teste estatístico Anova. O resultado da Anova apresentado na Tabela $6(\mathrm{~F}=0,706$; sig. $=0,621)$ indicou, ao nível de $5 \%$, a não existência de diferenças significativas entre os escores de imitação nesses setores. Para testar as possíveis diferenças nesses escores quanto ao setor, considerando-se separadamente as empresas pelo porte ( $2^{\mathrm{a}}$ fase), a amostra foi dividida por setores em três faixas de tamanho (pequenas, médias e grandes). Os resultados apresentados na Tabela 6 também indicam a não existência de possíveis diferenças entre os setores quando é considerado o porte das empresas.

Finalmente, as possíveis diferenças nos escores de imitação foram testadas quanto ao porte das empresas independentemente do setor de atuação ( $3^{\mathrm{a}}$ fase). Os resultados apresentados $(F=4,240 ;$ sig. $=0,020)$ na Tabela 6 indicam diferenças significativas ao nível de 5\% de significância. Sendo assim, a percepção dos gestores quanto à imitação varia segundo o porte da empresa. 


\section{CONCLUSÃO}

A presente pesquisa teve como objetivo identificar as dimensões que embasam o construto imitação entre empresas, com base na facilidade da comunicação e compreensão do conhecimento relevante utilizado nas competências das firmas, e propor um procedimento operacional para sua mensuração. Esse objetivo foi alcançado. Gerou-se exploratoriamente uma escala para a mensuração da imitação com base na percepção dos gestores sobre o regime tecnológico dos setores (MALERBA e ORSENIGO, 1993). Ainda, com base nos autovalores e dos escores fatoriais gerados para cada dimensão extraída, foi criado um índice para a mensuração da posição relativa das empresas em relação à imitação.

A escala desenvolvida possui validade de conteúdo pela sua inserção na rede teórica sobre o conceito imitação entre empresas; apresenta validade convergente, testada pela técnica estatística de análise fatorial exploratória; e apresenta consistência interna, caracterizada pela confiabilidade e unidimensionalidade das suas dimensões. $\mathrm{O}$ índice de imitação não apresentou diferenças significativas em relação aos setores investigados, mesmo considerando o porte das empresas. Interessantemente, ocorreram diferenças significativas quando se investigou a influência do porte independentemente dos setores. O que sugere, para os próximos estudos, a análise das variáveis que discriminam os grupos de porte.

A escala proposta é uma boa colaboração para a área, uma vez que a escala existente anteriormente para mensuração da imitação desenvolvida por Zander e Kogut (1995) apresenta problemas de validade de construto. No que se refere à validade de conteúdo, as dimensões cumulatividade e oportunidade do regime tecnológico não foram consideradas na sua elaboração, embora sejam notadamente influentes; não apresenta consistência interna uma vez que a unidimensionalidade de suas dimensões internas não foi testada; e também não apresenta validade convergente.

A escala proposta pode ser utilizada para mapear a imitação e relacioná-la com outras variáveis organizacionais, por exemplo, vinculadas ao desempenho ou estrutura. Também pode ser aplicada para identificar setores que apresentem maior ou menor grau de imitação, permitindo ao pesquisador escolher aqueles que forem os mais apropriados para seu objetivo. Futuramente, seria interessante verificar se existem grupos de setores semelhantes com relação à imitação, permitindo ao pesquisador e ao praticante da estratégia maior capacidade de generalização de seus achados. Em especial, o pesquisador teria maiores

Tabela 5 - Teste da homogeneidade da variância entre os grupos

\begin{tabular}{|l|c|c|}
\hline \multicolumn{1}{|c|}{ VARIÁVEL CATEGÓRICA } & ESTATÍSTICA DE LEVINE & SIGNIFICÂNCIA \\
\hline Setor de atuação & 0,208 & 0,812 \\
\hline Setor de atuação considerando pequenas empresas & 0,729 & 0,665 \\
\hline Setor de atuação considerando médias empresas & 0,583 & 0,783 \\
\hline Setor de atuação considerando grandes empresas & 0,210 & 0,141 \\
\hline Porte das empresas independentemente do setor & 0,850 & 0,519 \\
\hline
\end{tabular}

Tabela 6 - Resultados da Anova

\section{PORTE}

Setor de atuação

Setor de atuação considerando pequenas empresas

Setor de atuação considerando médias empresas

Setor de atuação considerando grandes empresas

Porte das empresas independentemente do setor

\section{ESTATISTICA F DE SNEDECOR}

0,706

SIGNIFICÂNCIA

0,912

1,299

1,699

4,240
0,621

0,553

0,287

0,211

0,020 
possibilidades de desenho de pesquisas, já que a multiplicidade de setores leva a uma capacidade de generalização limitada e o número de variáveis torna os estudos impraticáveis (PORTO e outros, 2009).

Do ponto de vista do praticante da estratégia, gera a possibilidade de identificar similaridades não aparentes entre os setores, norteando as ações estratégicas. Por exemplo, perfis ambientais similares em termos da imitação entre empresas em negócios relacionados podem permitir o reaproveitamento de experiências e competências com mais confiança ao longo do tempo e ampliar a possibilidade de economias de escopo intertemporais (HELFAT e EISENHARDT, 2004).

No entanto, a escala deve ser utilizada com cautela, pois os resultados são provenientes de uma amostra por conveniência que não representa os setores, restringindo-se, portanto, às empresas estudadas. Vale destacar ainda que, embora o processamento da análise fatorial tenha se mostrado adequado pelo índice Kaiser-Meyer-Olkin e pelo teste de esfericidade Bartlett, o tamanho da amostra para as 43 variáveis iniciais do estudo ficou abaixo do recomendado na literatura, ou seja, 2,6 respondentes por assertiva em vez de cinco. Também não foram testadas as validades discriminante e de critério. Essas limitações são aceitáveis já que o estudo aqui apresentado é exploratório. Esse primeiro panorama sugere que a escala proposta seja confirmada futuramente, mediante nova pesquisa que utilize uma abordagem probabilística e a técnica de análise fatorial confirmatória por meio de equações estruturais.

\section{REFERÊNCIAS}

BALESTRIN, A; VARGAS, L. M; FAYARD, P. Criação de conhecimento nas redes de cooperação interorganizacional. RAE-revista de administração de empresas, v. 45, n. 3, p. 52-64, 2005.

BARRETO, I; BADEN-FULLER, C. To conform or to perform? Mimetic behaviour, legitimacy-based groups and performance consequences. Journal of Management Studies, v. 43, n. 7, p. 1559-1581, 2006.

BATAGLIA, W; MEIRELLES, D. S. Population ecology and evolutionary economics: toward an integrative model. Management Research, v. 7, n. 2, p. 87-101, 2009.

CASSIOLATO, J. E. Interação, aprendizado e cooperação tecnológica. In: LUGONES, G. (Coord) Proyecto de revisión del manual de Bogotá. Bogotá, 2004. Disponível em: http://www.science.oas.org/ricyt/interior/ subredes\%5Cinnova\%5Cdocs/Cassiolato.pdf. Acesso em: 10.10.2009.
COHEN, J. Statistical power analysis for the behavioral sciences. 2nd ed. Hillsdale, NJ: Lawrence Erlbaum. 1988.

CUNHA, C. R; MELO, M. C. O. L. A confiança nos relacionamentos interorganizacionais: o campo da biotecnologia em análise. RAE-eletrônica, v. 5, n. 2, art. 18, 2006. Disponível em: http://wwwl6.fgv.br/rae/eletronica/ index.cfm?FuseAction $=$ Artigo $\& I D=3689 \&$ Secao $=$ ARTIGOS $\&$ Volume $=5 \&$ Numero=2\&Ano=2006. Acesso em 10.07.2008.

DELIOS, A; GAUR, A. S; MAKINO, S. The timing of international expansion: information, rivalry and imitation among Japanese firms, 1980-2002. Journal of Management Studies, v. 45, n. 1, p. 169-195, 2008.

DOBREV, S. D. Competing in the looking-glass market: imitation, resources, and crowding. Strategic Management Journal, v. 28, n. 13, p. 1267-1289, 2007.

DOSI, G; TEECE, D. Organizational competence and the boundaries of the firm. Berkeley: University of California at Berkeley, 1993. Working Paper n. 93-11.

ENKEL, E; GASSMANN, O. Creative imitation: exploring the case of cross-industry innovation. R\&D Management, v. 9999, n. 9999, 2010. Artigo publicado online antes da impressão. Disponível em: http://www3. interscience.wiley.com/journal/123294061/abstract. Acesso em 27.03 2010 .

ESTIVALETE, V. F. B; PEDROZO, E. A; CRUZ, L. B. The learning process in interorganizational relationship. BAR - Brazilian Administration Review, v. 5, n. 4, p. 319-331, 2008. Disponível em: http://anpad.org.br/periodicos/ arq_pdf/a_807.pdf. Acesso em 20.03.2009.

FEY, C. F; BIRKINSHAW, J. External sources of knowledge, governance mode, and R\&D performance. Journal of Management, v. 31, n. 4, p. $597-$ 621,2005

FREEMAN, C; SOETE, L. The Economics of Industrial Innovation. 3rd ed. Cambridge: MIT Press. 1997.

HAIR, J. F; BABIN, B; MONEY, A. H; SAMOUEL, P. Fundamentos de métodos de pesquisa em administração. Porto Alegre: Bookman. 2005.

HAUNSCHILD P. R; MINER, A. S. Modes of interorganizational imitation. Administrative Science Quarterly, v. 42, n. 3, p. 472-500, 1997.

HELFAT, C. E; EISENHARDT, K. M. Inter-temporal economies of scope, organizational modularity, and the dynamics of diversification. Strategic Management Journal, v. 25, n. 13, p. 1217-1233, 2004.

INGRAM, P. Interorganizational learning. In: BAUM, J. A. C. (Ed) The Blackwell Companion to Organizations. Maden, MA: Blackwell Publishing, 2005. p. 642-663. 
JANSEN, J. J. P; VAN DEN BOSCH, F. A. J; VOLBERDA, H. W. Exploratory innovation, exploitative innovation, and performance: effects of organizational antecedents and environmental moderators. Management Science, v. 52, n. 11, p. 1661-1674, 2006.

KIM, J; MINER, A. S. Vicarious learning from the failures and near-failures of others: evidence from the U.S. commercial banking industry. Academy of Management Journal, v. 50, n. 2, p. 687-714, 2007.

KIM, T; RHEE, M. Exploration and exploitation: internal variety and environmental dynamism. Strategic Organization, v. 7, n. 11, p. 11-41, 2009.

KOGUT, B; ZANDER, U. Knowledge of the firm, combinative capabilities, and replication of technology. Organization Science, v. 3, n. 3, p. 383397, 1992.

KOGUT, B; ZANDER, U. Knowledge of the firm and the evolutionary theory of multinational corporation. Journal of International Business Studies, v. 24, n. 4, p. 625-645, 1993.

LEVINTHAL, D. A; MARCH, J. G. The myopia of learning. Strategic Management Journal, v. 14, n. S2, p. 95-112, 1993.

LIEBERMAN, M; ASABA, S. Why do firms imitate each other? Academy of Management Review, v. 31, n. 2, p. 366-385, 2006.

MALERBA, F; ORSENIGO, L. Technological regimes and firm behavior. Industrial and Corporate Change, v. 2, n. 1, p. 45-71, 1993.

MARCH, J. G. Exploration and exploitation in organizational learning. Organization Science, v. 2, n. 1, p. 71-87, 1991.

MINER, A. S; RAGHAVAN, S. V. Interorganizational imitation: a hidden engine of selection. In: BAUM, J. A. C; MCKELVEY, B. (Eds). Variations in organization science: in honor of Donald T. Campbell. Thousand Oaks: Sage, 1999. p. 35-62.

NELSON, R. R; WINTER, S. G. An evolutionary theory of economic change. Cambridge: Harvard University Press, 1982.

NUNNALLY, J; BERNSTEIN, I. Psychometric theory. New York: McGrawHill, 1994.
PORTO, E. C; BRITO, L. A. L; SILVA, A. A; BATAGLIA, W; BRITO, E. Z. Ambientes organizacionais: uma proposta de classificação com uso de munificência, dinamismo e complexidade. BASE - Revista de Administração e Contabilidade da Unisinos, v. 6, n. 2, p. 101-119, 2009. Disponível em: http://www.base.unisinos.br/index.php?e=2\&s=9\&a=63. Acesso em 01.02.2010.

RACHID, A; BRESCIANI FILHO, E; GITAHY, L. Relações entre grandes e pequenas empresas de autopeças e a difusão de práticas de gestão da produção. Gestão \& Produção, v. 8, n. 3, p. 319-333, 2001.

ROGERS, E. M. Diffusion of innovations. 2nd ed. New York: Free Press, 1980.

ROGERS, E. M. Diffusion of innovations. 5th ed. New York: Free Press, 2003.

STABER, U. Imitation without interaction: how firms identify with clusters. Organization Studies, v. 31, n. 2, p. 153-174, 2010.

TEECE, D. J. Profiting from technological innovation: implications for integration, collaboration, licensing and public policy. Research Policy, v. 15, n. 6, p. 285-305, 1986.

TORRES, F. R; GUIMARÃES, T. A. Papel de visitas técnicas na aprendizagem e institucionalização de práticas de controle externo. BBR - Brazilian Business Review, v. 5, n. 1, p. 72-88, 2008. Disponível em: http://www. bbronline. com.br/artigos.asp?sess=det\&id=156. Acesso em 15.07.2009.

YAMAMURA, E; SONOBI, T; OTSUKA, K. Time path in innovation, imitation, and growth: the case of the motorcycle industry in postwar Japan. Journal of Evolutionary Economics, v. 15, n. 2, p. 169-186, 2005.

WANG, H; LI, J. Untangling the effects of overexploration and overexploitation on organizational performance: the moderating role of environmental dynamism. Journal of Management, v. 34, n. 5, p. 925951, 2008.

WINTER, S. Knowledge and competence as strategic assets. In: TEECE, D. (Ed) The competitive challenge: strategies for industrial innovation and renewal. Cambridge, MA: Ballinger, 1987. p. 159-164.

ZANDER, U; KOGUT, B. Knowledge and the speed of the transfer and imitation of organizational capabilities. Organization Science, v. 6, n. 1, p. 76-92, 1995. 